\title{
An ANT+ protocol based health care system
}

\author{
Nadeem Qaisar Mehmood \\ School of Science and Technology \\ University of Camerino \\ Italy \\ nadeemqaisar.mehmood@unicam.it
}

\author{
Rosario Culmone \\ School of Science and Technology \\ University of Camerino \\ Italy \\ rosario.culmone@unicam.it
}

\begin{abstract}
In remote health care Body Area Networks (BAN) are very popular but demand low energy consumption due to very constrained resources. For it several protocols, such as ZigBee, BlueTooth, WiFi etc, have been proposed but non has delivered the optimum results. These systems also demand vast interoperability among devices. Recently a propriety protocol ANT+ provides such features and strengthens the goals for Internet of Things (IOT). The authors describe a software architecture which flexibly integrates ANT+ protocol enabled sensors to deliver health care services. The approach is validated on a health care application that integrates heart rate, cadence, distance, foot steps and environmental temperature sensors. Described architecture is modular, flexible, scalable and possess several features.
\end{abstract}

Keywords-body networks, sensors, healthcare, ANT+, heart rate, footpod, temperature;

\section{INTRODUCTION}

The usage of wireless technology is evident in many disciplines, such as observation of lives of rare species [1], smart homes [2], medical assistance [3] and security surveillance [4]. Monitoring body parameters, through wireless Body Area Networks (BAN), is becoming important due to recent focus on healthcare to provide services, such as remote clinical care, diagnostics, monitoring, individual security, enhanced sports and fitness training applications [5]. It demands a wearable technology that serves to monitor the body parameters and can transmit wireless signals. Although wearable body sensors possess such valuable features but they also hold unavoidable constraints, such as optimum energy consumption, data exchange and cross domain message interpretation among nodes.

Wearable sensors are actually low power devices that operate in environments where monitoring operations are required for extended period of time and frequent battery replacements are not possible. This fundamental constraint has triggered many researchers to develop different Media Access Control (MAC) protocols with the objective of bandwidth maintenance and low energy consumption [6], [7], [8], [9].

A new legacy protocol ANT+ [10] is popular because of its robustness and optimum energy consumption in developing Wireless Personal Area Networks (WPAN), such as body parameter monitoring, smart homes, fitness sport services and gathering of the medical data with in the range of 20 meters. Its battery prevails upto 3 years comparitive to BLE and ZigBee with 1 year and 6 months respectively [11]. This ultra-low power wireless technology supports different kinds of low data rate network topologies, such as peer-to-peer, star or mesh. It establishes communication channels among sensor nodes transmitting signals at $2.4 \mathrm{Ghz}$ frequency. This protocol is being used in abundance with over 60 million deployed nodes. Such wireless devices are integratable only when they abide by ANT branded standards, defining communication formats, to support interoperability.

In order to provide general services the wireless device sensors are applied together in a group. Such integration is vital to achieve aggregate results and to apply mining mechanisms for prediction and description analysis. It has made the application developers much inflexible as they have to deal with different device drivers, ad-hoc APIs and noninterpretable different data formats. Every time a new device is added, from a different vendor in order to provide a new service, they have to rescan the previously written code to deal with different APIs and drivers. They demand a framework that would seamlessly integrate different devices following their APIs and would be able to exchange the data between the applications. The exchanged data and wireless devices must be interpretable across the wireless node boundaries after proper identification. The interoperability among devices demands interoperable data models, flexible dynamic device configuration and their smooth integration. Our application is an effort towards achiving such a flexible framework.

European Commission's ageing report [12] states that by 2060 one in three Europeans will be over 65 . The ratio of working people to the inactive, is shifting from 4 to 1 today to 2 to 1 by 2060. Information Communication Technology (ICT) can contribute, by providing European citizens with, better and cheaper services for health and ageing well. Healthcare sector, as a global market, forecasts to grow from 7.6 to 17.6 billion Euros by 2017 .

Remote healthcare services have taken care of the aging and young society, while utilizing telecommunication network and information technology. We have taken the advantage from the wearable computing devices [13], 
[10] and have developed an application in the domain of healthcare to monitor patients remotely for later descriptive and predictive analysis [14].

Our system is flexible, uses parametric approach to auto start the sensors with their configuration settings. It is scalable to add more sensors later. The system uses a modular approach but interprets the data messages from the different devices uniformly while using a single data interpreter module. Any user can build up his profile and uses it on his personal computer to monitor his body parameters, such as heart rate, cadence, distance, foot steps taken and environmental temperature. Our system stores the data locally on a collecting device and also securely share it remotely for the analysis purpose.

The remainder of this article is organized as follows. Section 2 is about ANT+ communication protocol. We discuss the developed system and its different features in section 3. It follows with a subsection with discussion about the system technical architecture. Interoperability and data storage features of the current system are also described in its subsections. The next, section 4, discusses the related work. A brief discussion about the future work plans is given in section 5, and in the last, section 6 gives a short summary.

\section{ANT PROTOCOL}

ANT is a proprietary protocol for applications where monitoring operations are desired for a long time with low battery consumption. It divides its bandwidth into 125 channels of width equal to $1 \mathrm{MHz}$ and operates on $2.4 \mathrm{GHz}$ spectrum with transmission duration less or equal to $150 \mathrm{mi}-$ crosecond/frame for 8 bytes of data. It uses special network keys to distinguish different networks [10]. ANT provides management of physical, data link, network and transport layers of OSI stack, see figure 1 [11]. However ANT+, an extension of ANT, manages session, presentation and application layers to provide data and devices interoperability. The format of information that ANT represents at the higher layers, if agreed upon by vendors and is defined mutually in a set, is actually ANT+. These definitions are called profiles and are typically tied to a specific use case, for example heart rate, blood pressure. ANT+ profiles allow both sides to understand each other, as both have already agreed upon a specific format [10].

ANT supports the establishment of numerous public or private managed networks. Each ANT network possess a unique identification. For two ANT devices to communicate they must be in the same network. There exists an ANT channel between two devices which must possess same frequency, message period, device type and transmission type (i.e. slave or master). Each node representing a sensor device, consists of an ANT protocol engine and a host Micro Controller Unit (MCU). It has both master and slave nodes which can participate in one or more networks [15]. There

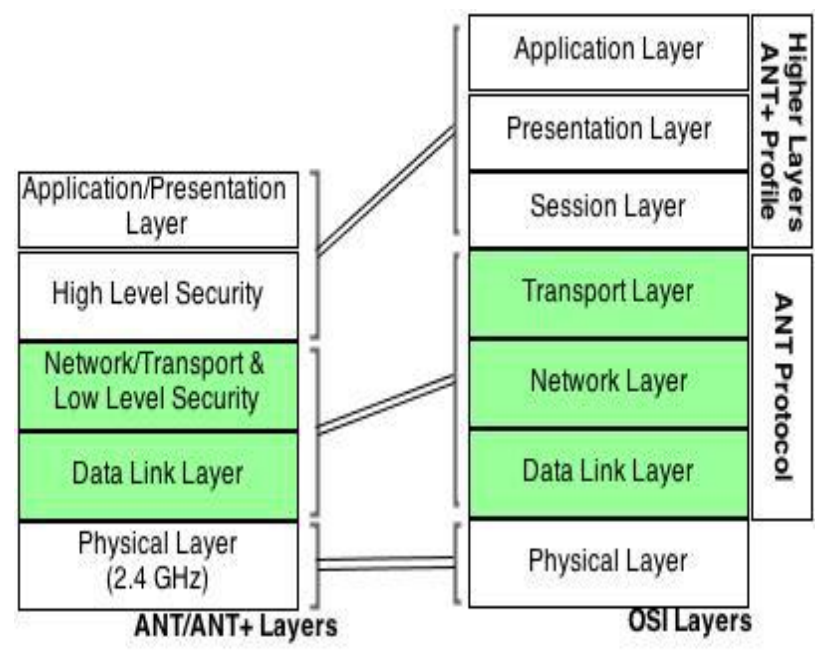

Figure 1. Correspondence between the ANT model, OSI layers and ANT+ profiles [11]

are three different ways for channel usage, i.e. independent, shared and scan channels [15].

ANT sends each of its 8 byte data packet over Radio Frequency (RF) channel using four different data types exclusively, such as broadcast, acknowledge, burst and advance burst [11]. Establishing a channel is an act of pairing two devices for communication through a process in which a slave obtains the complete unique channel ID of the master, it intends to communicate with, and agrees upon same frequency and message period. This relationship can be permanent, semi-permanent or transitory [15], [16].

The Flexible and Interoperable Data Transfer (FIT) protocol is designed specifically for data storage and share. It is defined for extensibility, interoperability and compactness of the information stored as a set of FIT message templates, such as user profiles, heart rate profiles and activity data in files. Such FIT files can be downloadable at a later time and any FIT-compliant device can interpret a FIT file from any other FIT-compliant device [17].

ANT File Share (ANT-FS) is an extension of the ANT protocol that provides a robust framework for transferring FIT files wirelessly between two ANT enabled nodes. It is often used in ultra low power display devices with storage capabilities, such as fitness watches. For security each ANTFS network possess a unique key [18].

\section{iII. Health Care System}

Managing disease and health issues is getting expensive worldwide. Sumarized research has shown that independent health management, exercise and physical monitoring has contributed greatly to prevent diseases and to improve health conditions. ANT Alliance has committedly provide simple and effective sensor devices that make the monitoring for health care easy and reliable [10]. 


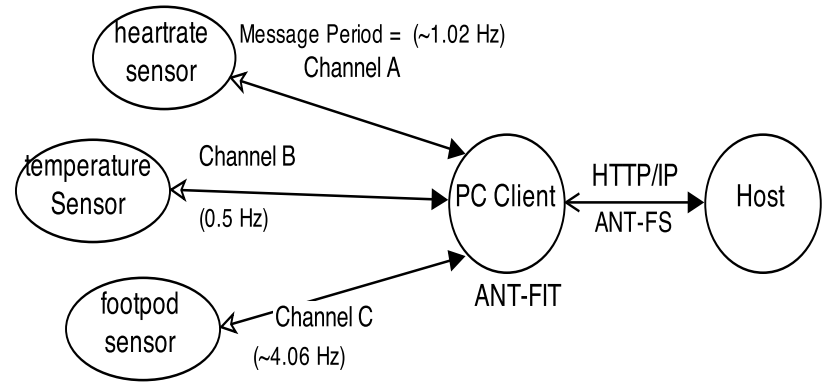

Figure 2. Wireless Sensor Nodes in the System network operating on 2.4 $\mathrm{GHz}$ frequency

There are around 22 million people effected with heart failure [19]. Carefull heart monitoring, regular exercises with better daily physical activity routine can strengthen the heart and cardiovascular system, improve circulation and lower the blood pressure. Weight, blood pressure, daily foot steps taken, bike speed during cycling, distance covered and calories burned are the core fundamental elements, that if managed carefully, will help in maintaining a healthy body. Such parameters contribute to achieve - healthy routine, timely dignostic decisions, medicose and medical checkups - hence leading towards a healthy society.

A system, body area network based, is developed that allows the connection of elderly people with their physicians and friends over the Internet. The resulting system uses automatic configurations, flexible architecture, desktop interface and wearable devices to acquire and deliver information about a person's wellbeing. The information can be selectively storeable and sharable, with other devices and people, using a secure technology.

Our system targets the heart patients and provides them with healthcare services. It uses ANT+ anabled sensors, such as heart rate, foot pod and temperature, to monitor a patient's body parameters and consolidates the information on a computer in FIT format files after encoding, please see figure 2. The unique network operates on $2.4 \mathrm{GHz}$ and each sensor plays the role of slave receiver only after establishing a secure communication channel. Each sensor operates at different channel configuration settings as depicted. The data collecting device, personal computer in current case, stores the data in FIT format files which is available for sharing with the host server. We discuss the system functionality as below.

Heart rate, or heart pulse, is the speed of the heart measured by the number of heartbeats per unit of time typically beats per minute (bpm). The system displays the speed of the heart or number of heartbeats, operating time and the previous heart rate of the user. It displays the current room temperature in Celsius (C) or Fahrenheit (F), minimum and maximum temperature since the device had started operation. It also uses the foot pod sensor to acquire

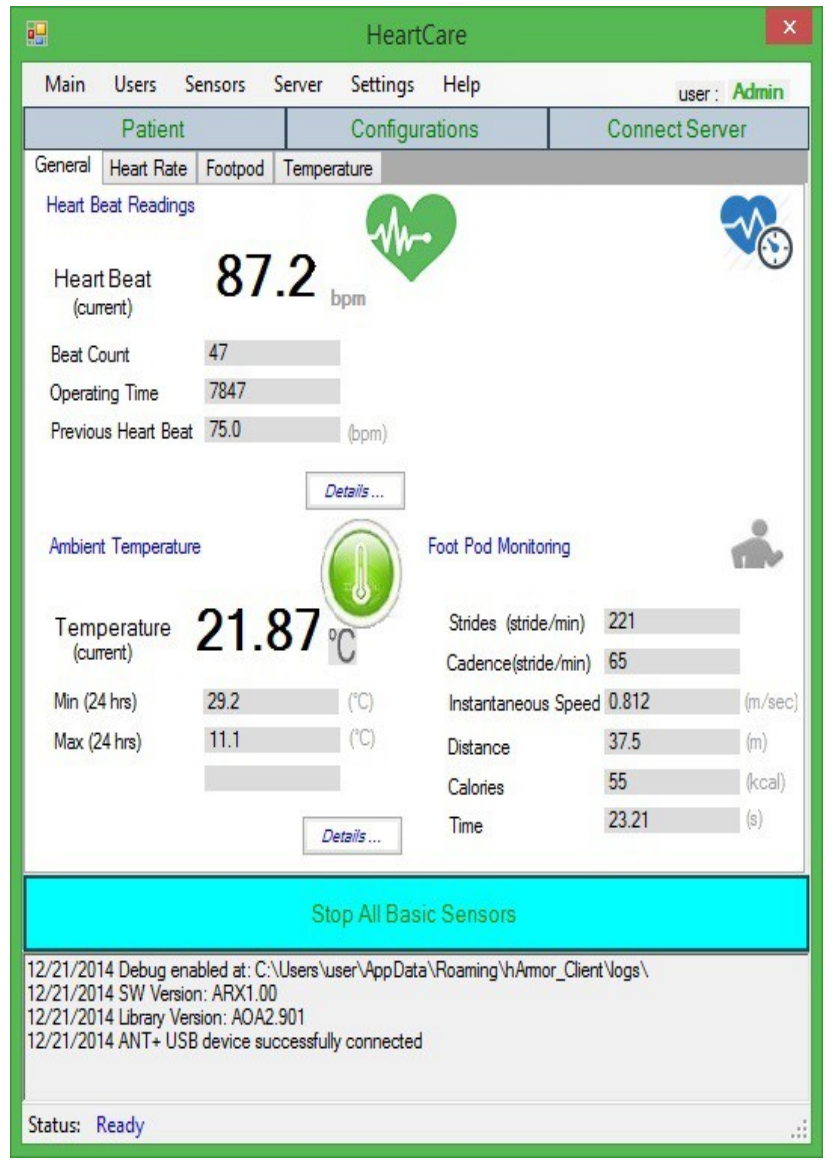

Figure 3. hArmor: Heart care monitoring application

information about the user's foot strides, cadence, distance covered (in meters), calories consumed, accumulated distance and strides count since the last battery change, please see figure 3 .

After performing authentication the system displays the current user and updates the user interface according to his rights. It also updates the application status based on the ANT+ protocol status. The different application statuses are: Closed, Ready, ANT-FS, Broadcast and Returned to Broadcast. System monitors ANT channels while displaying such statuses. It uses a parametrized approach to start up the application and to start sensor devices. These parameters, such as device configuration and application settings are stored in an XML file. A user can set the sensors to start automatically or can also start them all together manually. He can start and stop a single sensor individually. The application opens only required ANT+ communication channels, however the remaining channels stay close on the ANT+ enabled USB stick interface device which supports 8 channels in total. 


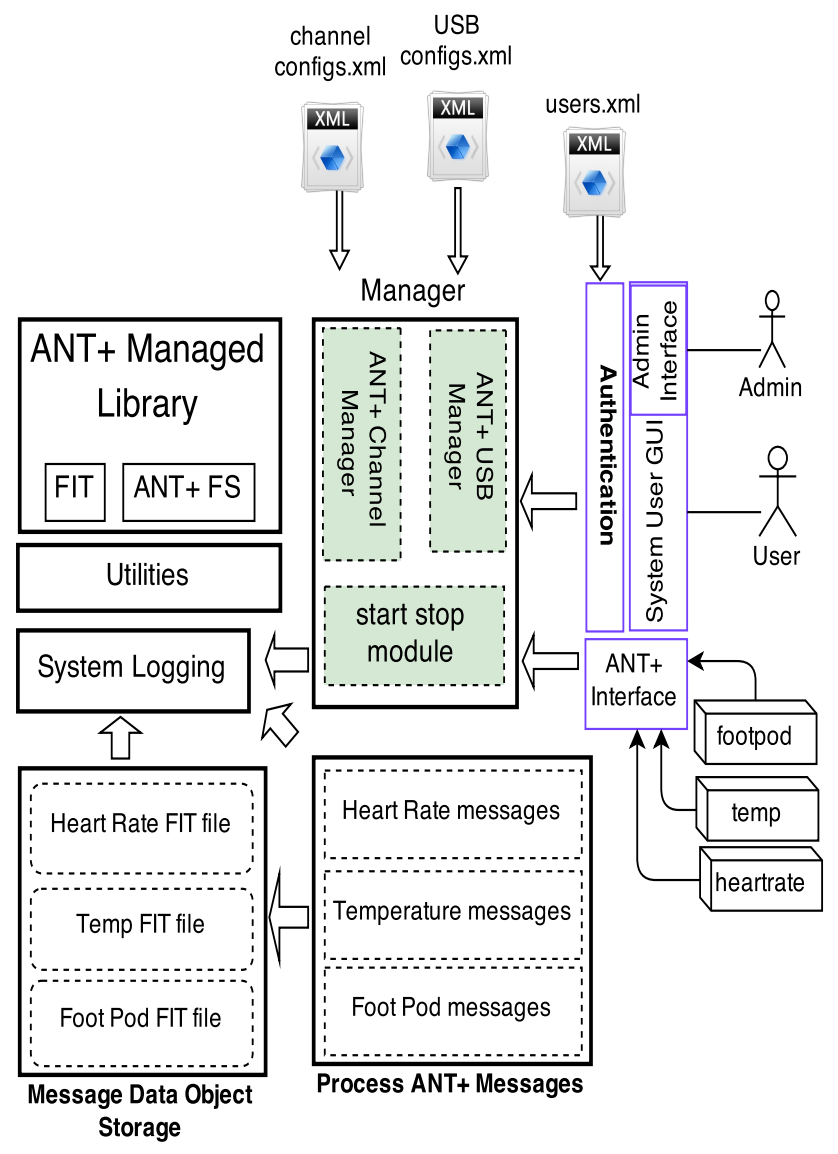

Figure 4. hArmor: System Modular Architecture

\section{A. Architecture}

The architecture of the implemented system, shown in figure 4, describes different modules and highlevel logical components with their relationships. The users interact with the system through the interface and can take advantage from the system services after secure authentication. Both normal and admin users have different levels of access rights. Each sensor communicates with the system through ANT+ enabled USB stick interface. The Manager module manages the ANT+ Universal Serial Bus (USB) stick and its associated different channels. This Manager module accepts the USB configurations from a structure format (an XML file) to configure and control an ANT+ enabled USB interface device.

Different sensors vary in configuration settings and communication message formats. Therefore, for each sensor device, the USB interface establishes a seperate ANT channel with appropriate message rate, Radio Frequency (RF), device type and ANT channel ID. A user can start and stop all or an individual sensor through the interface. The system supports auto start and stop feature for all the sensors. The system can load individual configuration parameters from an
XML file and can also use the default settings to establish an ANT channel with a sensor.

It is very important to distinguish and interpret each communication message at real time. This problem becomes even more complex when the system needs to communicate with more than one sensor device through a single interface. The system, on the runtime, has to check and understand message related information, a kind of meta data, such as device type, message data pages and message types of each upcomming message. ANT+ protocol supports more than 60 different message types and same is the case with the generated events. Although ANT+ managed libarary provides such a functionality to differentiate the messages based on their identification, but different sensors can generate same message ids, such as broadcast or acknowledge messages etc. Also we can not keep a totally seperate module for message interpretation and processing for each different device. Our application solves this problem by having a single static module for all the ANT+ messages, as depicted in figure 4. This module is further divided up into three smaller functions to distinguish and interpret an individual message based on the sensor device profile. ANT+ sensors send data in the form of data pages. Firstly, page definition is received which is followed by the page data itself. For each sensor device, the application manages both these message types and also interprets individual message bytes. It later stores their data in relevant temporary objects. We shall discuss the permanent data storage in the next coming sub section $\mathrm{C}$.

The system holds a logging module which records the debugging warnings and errors in textual files. The application also displays the $\log$ information within a small pannel, at the bottom of the interface, as shown in figure 3. The application possess a seperate Utilities module that caries small functions, such as time conversions or bit interpretations etc, available for all the modules. The system uses ANT+ managed libraries which also hold other libraries, such as ANT File Share (ANT-FS) and Dynastream's The Flexible and Interoperable Data Transfer (FIT) format extensions.

\section{B. Interoperability}

Information exchange is not possible between two parties unless both do not aggree upon establishing comminication and have ability to interpret eachother's messages. This fundamental constraint has put limitations on millions of sytems and devices to work coherently, especially when they are developed by different vendors. Interoperability, the ability of systems and devices to work together, is one major research goal in the domain of Information Communication and Technology (ICT). ANT+ protocol helps the system and devices to inter-operate, by implementing device profiles, to build up its ecosystem. Wirelessly transferred data that adheres to a given device profile will have the ability to interoperate with different devices from different manufac- 
turers if they also adhere to the same data format standard. For example an ANT+ heart rate sensor (using the ANT+ Heart Rate device profile) can be used with any ANT+ watch that also implements the ANT+ Heart Rate device profile. The heart rate sensor can be interchanged for any other ANT+ heart rate sensor, from any manufacturer. Our system also supports interoperability and can communicate with any vendor's heart rate, foot pod or temperature sensor, given the guarantee that device is enabled with an ANT+ module and is bond to the specific profile.

\section{Data Storage}

These days, systems require a storage structure that would be interoperable, compact, flexible and extensible. This is what ANT+ protocol provides with the help of its Flexible and Interoperable Data Transfer (FIT) protocol extension. Our application takes advantage of this feature and stores the sensor data permanently in FIT formated files. The system possess a message data storage module, as shown in figure 4, which stores individual sensor's data in the form of FIT format permanentaly. These files will be available for download by any device which have the rights to access and data decoding capability based on FIT format.

\section{RELATED WORK}

There are many systems which have been developed in healthcare domain, but we have not seen many which are developed using ANT+ protocol and have been made public. Following is some discussion about few related systems that use ANT+ technology.

The system in [20] is a biofeedback and gait analysis system for balance control for post stroke and gait related rehabilitation patients. They develop a wireless sensor network to provide home E-health care by integrating mobile technology and only two ANT+ sensors. However our system uses three sensors and monitors more than 8 features of the user. Our system possess a modular architecture with its interoperability and storage features, which make it distinguish and scalable.

In [21], the researchers develop a mobile based system which monitors the users who are mostly interested in sports. However our system is more focused on heart patients and uses parameters such as temperature, weight, distance travelled, cadence, footsteps taken and calories burned. Such features are not covered in [21]. We beleive that the factors which our system considers are more beneficial and meaningful for heart diseases. In [21] the system also uses a feedback approach taken from the doctors, we are also determined to extend such features in our system soon.

In [22], the authors have described the implementation of a WBAN for hip rehabilitation care called HipGuard, that uses ANT technology, however their entire focus is on hip patients.
In [23], the researchers have used different protocols to implement a system that focuses on getting mobile based data acquisition and provides feedback. Upto our knowledge their system uses sensor related to pedometer to measure footsteps, like us, but do not use heart rate and temperature sensors. They focus on blood pressure and blood glucose factors but use a different technology. Our system is different in context to interoperability, storage and above discussed health monitoring factors.

\section{Future WORK}

We aimed to extend our system in different ways. First of all we shall integrate other sensors into this architecture especially the blood pressure sensor. We are working on these factors and shall soon integrate such sensors to deliver more robust services. Secondly, we shall target more diseases in future. Study has shown that such a patient, with heart failure threats at some time in their life may also adopt other diseases, such as Alzheimer's, Asthma, Depression, Diabetes, Heart Failure and Obesity. Thirdly, we shall make the system flexible to upload whole data to a remote server for storage into more flexible and managable format for later predictive and descriptive analysis. Fourthly, we shall integrate the existing system with patient's caretakers such as doctors and relatives for realtime monitoring and feedback. Lastly, the same like service features will be developed for different mobile platforms.

\section{SUMMARY}

We have developed a system that facilitates a user to monitor and manage his health by observing his body parameters with the help of wearable technology and Body Area Wireless Networks. The system captures the heart rate, cadence, distance, foot steps taken and environmental temperature of a user to deliver health services. It integrates and encodes information into FIT files, such as heart rate, user profile etc. These FIT files are available for download to the other applications which have the access rights and decoding capability based on FIT data format. Such applications can store this data into different format for predictive and descriptive analysis. The system is under development and possess a very flexible architecture to integrate more sensors to deliver more robust services. It uses an automatic parametric approach to buildup a flexible and scalable architecture.

\section{REFERENCES}

[1] A. Mainwaring, D. Culler, J. Polastre, R. Szewczyk, and J. Anderson, "Wireless sensor networks for habitat monitoring," in Proceedings of the 1st ACM International Workshop on Wireless SensorNetworks and Applications, ser. WSNA '02. New York, NY, USA: ACM, 2002, pp. 88-97. [Online]. Available: http://doi.acm.org/10.1145/570738.570751 
[2] D. Surie, O. Laguionie, and T. Pederson, "Wireless sensor networking of everyday objects in a smart home environment," in Proceedings of the International Conference on Intelligent Sensors, Sensor Networks and Information Processing (ISSNIP), Ume University, Sweden. IEEE, 2008, pp. 189-194.

[3] "An overview of telecare and telehealth : Headline findings," UK Department of Health, Whole Systems Demonstrators (WSD), Tech. Rep., Sep 2013.

[4] N. Grang and A. Gupta, "Wireless sensors network: An overview," International Journal of Modern Computer Science (IJMCS), April 2013.

[5] M. Chen, S. Gonzalez, A. Vasilakos, H. Cao, and V. C. Leung, "Body area networks: A survey," Mobile Network Applications, vol. 16, no. 2, pp. 171-193, April 2011.

[6] IEEE 802.15.4 Strandard on Wireless LAN Medium Access Control (MAC) an Physical Layer (PHY) specifications for low Rate Wireless Personal Area Networks, Institute Electrical Electronics Engineers (IEEE) Std., 2006. [Online]. Available: http://standards.ieee.org/about/get/802/802.15.html

[7] J. Polastre, J. Hill, and D. Culler, "Versatile low power media access for wireless sensor networks," in Proceedings of the 2nd International Conference on Embedded Networked Sensor Systems, ser. SenSys '04. New York, NY, USA: ACM, 2004, pp. 95-107.

[8] W. Ye, J. Heidemann, and D. Estrin, "An energyefficient mac protocol for wireless sensor networks," in Proceedings of the IEEE Infocom:Twenty-First Annual Joint Conferenceof the IEEE Computer and Communications Societies, USC/Information Sciences Institute. New York, NY, USA: IEEE, June 2002, pp. 1567-1576. [Online]. Available: http://dx.doi.org/10.1109/INFCOM.2002.1019408

[9] — "Medium access control with coordinated adaptive sleeping for wireless sensor networks," IEEE/ACM Transactions on Networking, vol. 12, no. 3, pp. 493-506, jun 2004.

[10] Thisisant: the wireless sensor network solution. Dynastream Innovations Inc. [Online]. Available: http://www.thisisant.com

[11] S. Khssibi, H. Idoudi, A. V. D. Bossche, and L. A. Val, Thierryand Saidane, "Presentation and analysis of a new technology for low-power wirelesssensor network," International Journal of Digital Information and Wireless Communications (IJDIWC), vol. 3, no. 1, pp. 75-86, 2013. [Online]. Available: http://sdiwc.net/digitallibrary/web-admin/upload-pdf/00000591.pdf

[12] Editor, "The 2012 ageing report: Economic and budgetary projections for the 27 eu member states (2010-2060)," European Commission, Tech. Rep., 2012. [Online]. Available: https://ec.europa.eu/digital-agenda/en

[13] Body sensor networks. Imperial College London. [Online]. Available: http://ubimon.doc.ic.ac.uk/bsn/m621.html

[14] P.-N. Tan, M. Steinbach, and V. Kumar, Introduction to Data Mining, (First Edition). Boston, MA, USA: Addison-Wesley Longman Publishing Co., Inc., 2005.
[15] "Ant message protocol and usage: Application notes," Dynastream Innovations Inc., online doc, Jul 2007. [Online]. Available: www.thisisant.com

[16] "Ant device pairing : Application notes," Dynastream Innovations Inc., Tech. Rep., Mar 2013. [Online]. Available: www.thisisant.com

[17] "Flexible and interoperable data transfer (fit) protocol," Dynastream Innovations Inc., online doc, August 2014. [Online]. Available: http://www.thisisant.com/resources/fit

[18] "Ant file share (ant-fs) technical specification," Dynastream Innovations Inc., online doc, January 2012. [Online]. Available: http://www.thisisant.com/resources/antfs-technical-specification

[19] Thisisant: The facts. Dynastream Innovations Inc. [Online]. Available: http://www.thisisant.com/business/why-ant/facts

[20] A. Johansson, W. Shen, and Y. Xu, "An ant based wireless body sensor biofeedback network for medical e-health care," in Wireless Communications, Networking and Mobile Computing (WiCOM), 2011 7th International Conference on, Sept 2011, pp. 1-5.

[21] A. Baca, P. Kornfeind, E. Preuschl, S. Bichler, M. Tampier, and H. Novatchkov, "A server-based mobile coaching system,” Sensors, vol. 10, pp. 10640-10 662, 2010.

[22] M. Soini, J. Nummela, P. Oksa, L. Ukkonen, and L. Sydnheimo, "Wireless body area network for hip rehabilitation system," Ubiquitous Computing and Communication Journal 3.5, 2008.

[23] M. Strasser, E. Helm, A. Schuler, M. Fuschlberger, and B. Altendorfer, "Mobile access to healthcare monitoring data for patients and medical personnel," in 24th International Conference of the European Federation for Medical Informatics Quality of Life through Quality of Information, 2012. 